\title{
BMJ Open Video-based smartphone app ('VIDEA bewegt') for physical activity support in German adults: a study protocol for a single-armed observational study
}

\author{
Tillmann Fischer (D , ${ }^{1}$ Paul Stumpf, ${ }^{1}$ Gesine Reinhardt, ${ }^{1}$ Peter E H Schwarz, ${ }^{1,2}$ \\ Patrick Timpel ${ }^{1}$
}

To cite: Fischer T, Stumpf $P$, Reinhardt G, et al. Videobased smartphone app ('VIDEA bewegt') for physical activity support in German adults: a study protocol for a single-armed observational study. BMJ Open 2020;10:e034027. doi:10.1136/ bmjopen-2019-034027

- Prepublication history and additional material for this paper are available online. To view these files, please visit the journal online (http://dx.doi. org/10.1136/bmjopen-2019034027).

TF, PS and GR are joint first authors.

Received 02 September 2019 Revised 06 December 2019 Accepted 22 January 2020

Check for updates

(C) Author(s) (or their employer(s)) 2020. Re-use permitted under CC BY-NC. No commercial re-use. See rights and permissions. Published by BMJ.

${ }^{1}$ Department for Prevention and Care of Diabetes, Department of Medicine III, Faculty of Medicine Carl Gustav Carus, Technische Universität Dresden, Dresden, Germany

${ }^{2}$ Paul Langerhans Institute Dresden of the Helmholtz Center Munich at University Hospital and Faculty of Medicine,

Technische Universität Dresden, Dresden, Germany

Correspondence to

Tillmann Fischer;

tillmann.fischer@tu-dresden.de

\section{ABSTRACT}

Introduction Insufficient physical activity is one of the most important risk factors for non-communicable diseases. Physical activity should therefore be intensively promoted in all age groups. Several trials suggest that it can be effectively increased through smartphone interventions.

However, few of the smartphone-interventions available on the market have been scientifically evaluated. Therefore, the described study aims to assess the short-term and long-term effects of the smartphone intervention 'VIDEA bewegt' to increase physical activity. The trial is designed as a single-armed observational trial to assess effects under real-life conditions.

Methods and analysis The intervention consists of the smartphone-application 'VIDEA bewegt', which is a video-based preventative programme to improve physical activity in everyday life. The application contains several features and components including educational videos, documentation of activity and motivational exercises. A sample size of at least 106 participants is aimed for. The primary objective of this study is to determine the effect of the application on physical activity in German adults. Secondary objectives are to evaluate the selfefficacy, health-related quality of life and usability of 'VIDEA bewegt'.

Data collection is based on online questionnaires, as well as system-internal recorded data.

Changes of outcomes from baseline to programme completion and follow-up will be calculated.

Ethics and dissemination The Ethics Committee of the Technical University Dresden approved the study on 25 May 2019 (EK 272062019). All data are processed anonymously and stored on servers only accessible by authorised personnel. The results of the study and the results of the usability test are aimed to be published in a scientific journal.

Trial registration number German Clinical Trials Registry (DRKS00017392).

\section{INTRODUCTION}

\section{Background}

Insufficient physical activity is considered to be one of the most important risk factors for non-communicable diseases along with
Strengths and limitations of this study

- The evaluation of the intervention is carried out under real-life conditions.

Various approaches are used to describe the effects of the intervention.

- Since participation in the evaluation is voluntary, inclusion criteria for participation are broad and there is a risk of selection bias. Due to the multilayered structure of the intervention, it will differ for each individual user. Furthermore, most data collected is based on self-assessment.

tobacco, alcohol consumption and unhealthy diet. ${ }^{1}$ Being defined as less than $150 \mathrm{~min}$ of moderate activity, 75 min of vigorous activity or less than 600 metabolic equivalent (MET) minutes per week, ${ }^{2}$ its prevalence for both sexes is $27.5 \%$ worldwide and $42.2 \%$ in Germany. ${ }^{3}$ As activity progressively decreases with age, it is likely to become a growing problem in our ageing population. ${ }^{4}$ In order to prevent chronic diseases and to minimise the risk of premature death, physical activity should therefore be intensively promoted in all age groups. ${ }^{5-7}$

The widespread availability of smartphones has increased the interest in exploring the potential of digitisation in preventative healthcare. $^{8}$ Digital interventions can be made available without great cost and effort ${ }^{9}$ and could help to provide healthcare for those with limited access to it. ${ }^{10}$ The potential of such interventions is demonstrated by the fact that more than $80 \%$ of German citizens aged 14 or older, and already more than $40 \%$ of those aged 65 or older, were using a smartphone regularly in 2017. ${ }^{11}$ Focusing on physical activity, several scientific trials suggest that it can be effectively increased through smartphone interventions, which provide information and performance-related feedback, 
monitor behaviour change, enable social networking and goal setting and include motivational messages or reminders. ${ }^{12-15}$ Although the number of health apps on the market is already high (in $2016>80000$ apps in categories 'health and fitness' and 'medicine ${ }^{16}$ ), and their potential in the health sector is promising, ${ }^{9} 1718$ only few of these apps use evidence-based content, practices and features. ${ }^{19}$ Developing digital interventions that work equally well for people with different expectations, opportunities and cultural backgrounds is challenging. ${ }^{20}$

A major limitation of past studies is the fact that they use apps which are specifically developed for the purpose of the study and not commercially available. ${ }^{21} 22$ As a consequence, only few of the apps available on the market have been scientifically evaluated, ${ }^{23}{ }^{24}$ although an evaluation could benefit their clinical use. ${ }^{25}$ Various methodological challenges of evaluating digital devices using randomised controlled trials are known. These challenges include randomisation, timing of assessment, acceptance by patients and physicians, blinding, as well as defining control groups and relevant endpoints. ${ }^{26}$ Apart from that, many digital health interventions combine different intervention components (tracking of health data, motivating messages based on individual goals, performancerelated feedback, etc) and can therefore differ in their effects on individuals-despite identical utilisation. This modular structure of digital applications and the associated flexibility and customisability are great strengths of those interventions, ${ }^{27}$ but make it difficult to conduct randomised controlled trials, as recommended by several studies. ${ }^{19} 28$

There is a need for mid-term and long-term data on the effectiveness and safety of such dynamic smartphoneapplications. ${ }^{28}{ }^{29}$ In addition, the time course of intervention effects and the strategies needed to achieve sustainable change have not yet been fully investigated. ${ }^{12}$ An iterative and user-centred development is regarded as key to identify needs and preferences of the relevant target group, ${ }^{30-33}$ and overcome barriers of prototypes in early development stages. ${ }^{34}$

\section{Objective}

The overall objective of this study is to assess the effect of the video-based smartphone app 'VIDEA bewegt' on physical activity in German adults.

Secondary objectives are to evaluate self-efficacy, healthrelated quality of life and usability of 'VIDEA bewegt', especially with regards to participants' satisfaction with the app.

\section{Hypotheses}

- The users of 'VIDEA bewegt' who participate in the study increase their average daily step count and achieve a higher number of MET minutes per week, a significantly higher health-related quality of life, a significantly higher motivational, maintenance and recovery self-efficacy after the first 4 weeks and after completion of the 8-week course, compared with the beginning of data collection.

- The above-mentioned changes will last through the follow-up period $(2 / 4 / 6 / 12$ months after the completion of the programme). The strength of the effects is influenced by individual background (eg, educational background, socioeconomic status) and by the participants' use of the app.

\section{Trial design}

The evaluation of the app 'VIDEA bewegt' is designed as a single-arm observational study, assessing the app's effectiveness under real-life conditions. Additionally, think aloud interviews are conducted beforehand within the framework of a usability test to integrate user-oriented design.

\section{METHODS: PARTICIPANTS, INTERVENTIONS AND OUTCOMES Study dates}

The study was developed from October 2018 to April 2019. The ethics proposal and the prestudy with usability and pretest were carried out from January 2019 to May 2019. Data collection started in July 2019. Depending on sufficient numbers of participants, it is expected to be continued until December 2019 for a first evaluation of the effects during programme use. The follow-up is planned to be continued until the end of 2020 .

\section{Study setting}

'VIDEA bewegt' is an app enabling users to access educational content via their smartphone anywhere and at any time. 'VIDEA bewegt' was officially certified as a physical activity course and is covered by some health insurances. It was made available on the German market for Android and iOS in March 2019. More information can be found on the German website. ${ }^{35}$

A clinical visit or in-person contact with a physician or diabetes specialist is not required. However, it is possible to consult experts in preventative healthcare and sports science via an integrated chat function. Problems can also be discussed with other users in a forum.

As the developer of 'VIDEA bewegt', the TUMAINI Institut für Präventionsmanagement $\mathrm{GmbH}$ is responsible for the overall functioning of the app.

\section{Eligibility criteria}

All users of 'VIDEA bewegt' are potential participants of the study and will be invited to take part in the evaluation after registration in the app. Inclusion criteria for participation in the study are the active use of 'VIDEA bewegt' and informed consent to participate in the study (figure 1). In order to complete the app-registration, users must be of legal age ( $\geq 18$ years old), agree to the terms of the app, confirm that physical contraindications such as musculoskeletal conditions, cardiovascular diseases and cancer do not apply, and speak and write German fluently. Inclusion and exclusion are not restricted by 


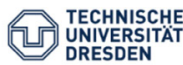

000

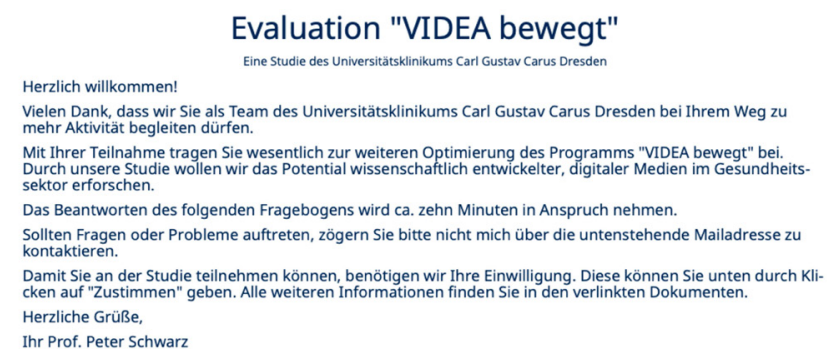

E-Mail: tillmann.fischer@tu-dresden.de

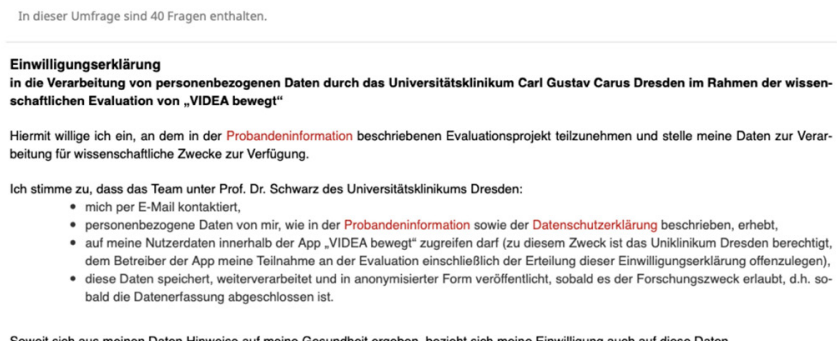

Akzeptieren

Figure 1 Informed consent. The informed consent is presented to the participants as the starting page of the first online questionnaire. After the introduction text the privacy policy will be explained. Afterwards, consent to participate in the study will be given.

other criteria such as health or age. Eligibility criteria are based on self-assessment.

\section{Patient and public involvement}

Potential participants were included in the study development in order to make methods understandable and functional, to better understand observed effects and to gather errors and problems to serve future projects. One step in this process involved pretesting the questionnaires in order to assess their logic and understandability. Another important step was the usability test which included interviews with selected individuals. Possibilities to improve the app could be demonstrated.

In the context of actual app usage and study participation, participants are involved in system improvement by recording app usage problems in questionnaires.

\section{Interventions}

'VIDEA bewegt' is a preventative programme to improve physical activity in everyday life and strength endurance. It was developed in cooperation with physicians of the university hospital Dresden. The programme uses videos to present content and exercises and aims to motivate users to increase physical activity on a regular basis (see online supplementary fiugres 1-5).
It consists of eight 45 min stages, each representing one course week including four videos (online supplementary figure 3 - stage structure). Each week, a new stage will be unlocked in ascending order. Therefore, the minimum time needed to complete the programme is 7 weeks and 1 day. The maximum period for completing the programme is 1 year.

App users are free to decide when and how intensively they want to use the programme. The app has a multimodular structure and contains the following features and components:

- Educational videos.

- Synchronising steps.

- Calculating and displaying activity minutes.

- Motivational exercises under 'My Focus'.

- Motivational messages.

- Answering quiz questions.

- Chat/forum.

\section{Educational videos}

In these videos, an expert in preventative healthcare and an expert in sports science provide both theoretical medical content and practical exercises to participate in. The theoretical videos have a length of 2-4 min and the exercise videos each last approximately $20 \mathrm{~min}$. In total there are 32 videos and 17 additional videos. In the first video of each stage, users receive theoretical information regarding physical activity and its effects on body and mind. For example, the potential negative effects of a physically inactive lifestyle are explained together with strategies for their prevention. The second video presents exercise instructions for endurance and strength exercises. The aim of these videos is for users to get to know the exercises so that they can perform them on their own. The third video refers to the behavioural change model of Greaves $e t a l^{86}$ to build up motivation to increase activity through techniques of motivational psychology applied practically in the category 'My Focus'. The fourth video contains practical recommendations on how exercise can be integrated into everyday life with the help of different types of exercise. Add-on videos will be made available on topics like barefoot walking, sauna visiting and healthy diet.

\section{Synchronising steps}

In order to track activity, the app offers the opportunity to synchronise step numbers on a daily basis (online supplementary figure 4 - Activity). Users can either enter the step numbers into the app themselves, if they are using an external device, or synchronise their steps automatically. For automatic synchronisation, 'VIDEA bewegt' is connected with a pedometer app on the phone.

\section{Calculating and displaying activity minutes}

When registering, users select an average activity level of $<30 \mathrm{~min}, 30-60 \mathrm{~min}$ and $>60 \mathrm{~min} /$ day to set a daily activity goal (online supplementary figure 4). Physical activities such as cycling, swimming, housework can 
Table 1 Overview of the measures used

\begin{tabular}{|c|c|c|c|}
\hline Measure & Topic & Description & $\begin{array}{l}\text { Item } \\
\text { number }\end{array}$ \\
\hline GPAQ $^{6364}$ & Physical activity & $\begin{array}{l}\text { The Global Physical Activity Questionnaire (GPAQ) measures the } \\
\text { intensity and duration of physical activity at work, during transport } \\
\text { and during leisure time. The data are quantified by a conversion into } \\
\text { metabolic equivalent (MET) minutes per week. One MET represents } \\
\text { the energy consumption at rest. The GPAQ defines } 4 \text { METs for } \\
\text { moderate activity and } 8 \text { METs for vigorous activity. }{ }^{65}\end{array}$ & 16 \\
\hline SF- $-8^{6667}$ & $\begin{array}{l}\text { Health related } \\
\text { quality of life }\end{array}$ & $\begin{array}{l}\text { The SF-8 (Short Form Health Survey } 8 \text { ) covers the eight dimensions } \\
\text { of SF-36 on the basis of eight questions: physical functioning, role- } \\
\text { physical, bodily pain, general health, vitality, social functioning, role- } \\
\text { emotional and mental health. }\end{array}$ & 8 \\
\hline RecSE, ${ }^{68}$ adapted to ${ }^{69}$ & $\begin{array}{l}\text { Recovery self- } \\
\text { efficacy }\end{array}$ & $\begin{array}{l}\text { Self-efficacy, related to the return to activity after a setback, is } \\
\text { estimated on the basis of three items. }\end{array}$ & 3 \\
\hline $\begin{array}{l}\text { AppEx-Mod } \\
\text { (self-constructed) }\end{array}$ & General information & $\begin{array}{l}\text { Shortened version of the App-External Questionnaire (AppEx): } \\
\text { questions about weight, height, endurance and muscle strength, } \\
\text { participation in further courses. }\end{array}$ & 5 \\
\hline $\begin{array}{l}\text { Peva-FB } \\
\text { UEQ }^{70}\end{array}$ & Process evaluation & $\begin{array}{l}\text { A series of author-constructed questions to assess satisfaction } \\
\text { with individual components of the programme and user behaviour. } \\
\text { Additional integration of UEQ-S (User Experience Questionnaire } \\
\text { Short), which assesses the user-friendliness of a product using eight } \\
\text { opposing pairs of characteristics. }\end{array}$ & 5 \\
\hline
\end{tabular}

be documented by selecting from a list and entering a number of minutes. In addition, the collected step numbers and training sessions contribute to the activity goal.

\section{Motivational exercises}

Based on contents of the third video of each stage, a new section in the app category 'My Focus' will be unlocked each course week, enabling users to express their thoughts in text fields for self-reflection and motivation. Sections of 'My Focus' include the user's personal motive, possible action plans, power sources and strengths, social support, rewards, stopping negative thoughts, if-then plans and dealing with setbacks.

\section{Motivational messages}

Motivational messages appear each time the app is opened. These messages contain a review of the previous video, the reminder for a specific motivational exercise under 'My Focus' and a preview of the upcoming content. As such, the motivational messages are individualised using the aspects of the motivational exercises, which were initially selected by the patients themselves.

\section{Answering quiz questions}

At the end of each stage, two to three multiple-choice questions will be displayed on the content of the videos, primarily to review recently received information. Feedback is provided on given answers, yet accuracy does not influence the further programme.

\section{Chat/forum}

The 'Exchange' app category offers two features to users with questions or problems (online supplementary figure 5). Experts can be contacted directly in a chat. Furthermore, a forum is included to discuss questions on the topics 'Questions and discussions about the course', 'Smalltalk', 'Technology and support' with other users.

\section{Measurement of outcomes}

Primary outcome: physical activity

- Step numbers/minutes of activity.

- Mean MET minutes per week.

\section{Secondary outcomes}

- Self-efficacy (motivational, maintenance, recovery self-efficacy). 


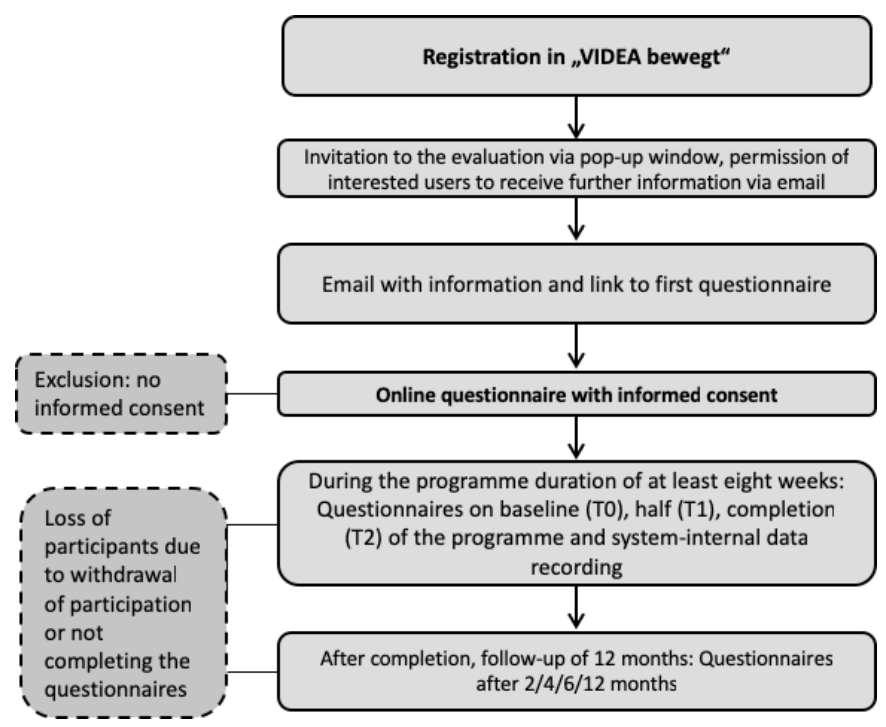

Figure 2 Participant timeline.

- Health-related quality of life: Mental Health Component Summary score and Physical Health Component Summary score.

- User behaviour and obstacles to the use of the programme.

See table 1 for detailed information.

\section{Participant timeline}

The evaluation will begin with people registering in the 'VIDEA bewegt' app without knowing anything about

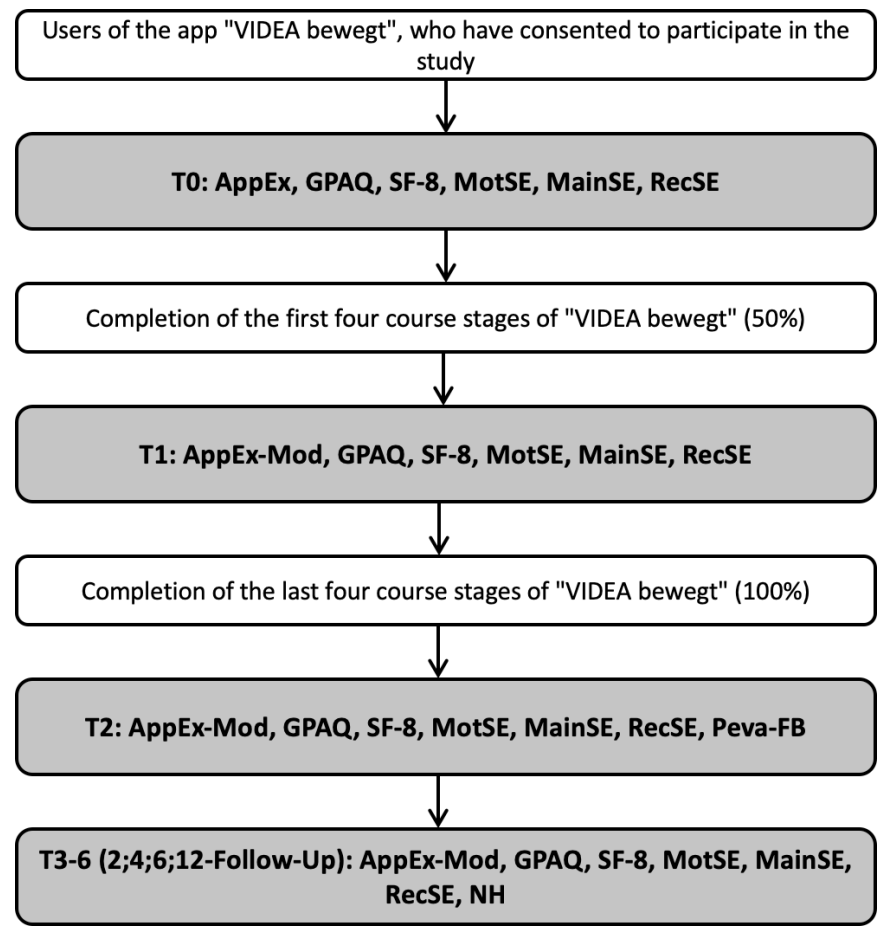

Figure 3 Flow diagram. AppEx, App-External Questionnaire; GPAQ, Global Physical Activity Questionnaire; MainSE, maintenance self-efficacy; MotSE, motivational self-efficacy; $\mathrm{NH}$, sustainability; Peva-FB, process evaluation; RecSE, recovery self-efficacy; SF-8, Short Form Health Survey 8. this study (figure 2). Once registration is complete, all users of the app will be invited to participate in the study via a pop-up window. Further information as well as the link to the first questionnaire will be sent by e-mail. Data are collected using a total of seven online questionnaires (figure 3). After completion of fourth stage and eighth stage, selected system-internal data, will also be extracted from 'VIDEA bewegt'.

\section{Sample size}

In similar projects, which were conducted as randomised controlled trials, the total number of participants ranged from 40 to $361 .{ }^{9153738}$

In the SMART MOVE study, for example, 90 participants were divided into two groups. ${ }^{15}$ After an a priori power analysis using $\mathrm{G}^{*}$ Power $^{39}$ with an assumed small to medium effect size for change of self-efficacy as well as for change of physical activity ${ }^{40-42}$ a necessary sample size of at least 27 was calculated for the t-tests. Starting from $20 \%$ study or intervention drop-out (conservative assumption), a total of $\mathrm{n}=33$ participants will have to be recruited. In order to assess the influence of potential confounders such as age or period of use, a multiple regression analysis is planned. Therefore, a larger sample size of at least 106 persons is aimed for. ${ }^{434}$

\section{Recruitment}

Users of 'VIDEA bewegt' will be invited to the study after registration via a popup-window. Since two health insurance companies were involved in the development of the app, people insured with these health insurance companies can use the app free of charge. For members of other health insurance companies, a fee is due. In order to recruit further study participants, a fee waiver will be offered on the condition of participation.

Study participation will be compensated with $€ 15$. Consent to participate in the study will be given at the beginning of the first questionnaire (figure 1). Participants will receive e-mails containing a link to access each questionnaire at the relevant stage. If no reply was received within 2 days, a reminder e-mail will be sent.

\section{METHODS: DATA COLLECTION, MANAGEMENT AND ANALYSIS Usability test}

A usability test was carried out before starting data collection. For this usability test, the think aloud method was used, which is common when developing user centred designs. ${ }^{45}$ Think aloud describes an interview in which, for example, an app is tested, and the test persons are asked to express their thoughts and impressions, particularly concerning obstacles they encounter when using the app, verbally. This approach provides an insight into strengths and weaknesses of a system. ${ }^{46}$ Think-aloud methods are a robust tool to identify about three quarters of the usability problems with the help of only four to five test persons. ${ }^{47}$ 
Ten test persons were recruited to work through the structure of the app while aiming to achieve various specified goals (eg, input their daily step count). They were asked to describe their thoughts, impressions and problems verbally. The audio track was recorded along with the screen of the smartphone. The recorded interview was transcribed and analysed according to Mayring. ${ }^{48}$ These insights in the usability of 'VIDEA bewegt' were used to further improve the programme's usability. Thereby, the effectiveness of 'VIDEA bewegt' will be less likely to be confounded by usability problems.

\section{Pretest of questionnaires}

The questionnaires used in the evaluation contain both validated and non-validated questions constructed by the authors. The questionnaires were analysed with the help of expert opinions regarding their structure and selectivity. The non-validated questions were tested in a think aloud test and optimised on the basis of these results.

Apart from that, a pretest of the complete questionnaires was conducted by sending a link to a test questionnaire to 20 people. They were asked to record the time needed to answer the questionnaire and to comment on spelling, grammar, comprehensibility, presentation, structure and logic of the questions.

\section{Evaluation}

The actual study is based on two essential data sets. (1) Data gathered via the online questionnaires is stored in SURVEY (based on Limesurvey) and (2) system-internal data using step numbers and activity minutes (see table 2).

The questionnaires use internationally established scores for physical activity, health-related quality of life and selfefficacy (see table 1 and online supplementary file).

Plans to promote participant retention and complete follow-up Subjects who withdraw their study participation will receive a final questionnaire for process evaluation. Data from respondents who withdraw or stop completing questionnaires will be included as long as questionnaires were completed at least at two different stages.

Table 2 System-internal data

\begin{tabular}{|c|c|}
\hline General & $\begin{array}{l}\text { Email address } \\
\text { Day of registration+day of } \\
\text { completion health insurance } \\
\text { company }\end{array}$ \\
\hline User behaviour & $\begin{array}{l}\text { Date/time at which a video was viewed } \\
\text { Active use of expert chat } \\
\text { Active use of forum }\end{array}$ \\
\hline Documented data & $\begin{array}{l}\text { Activity level }<30 \text { min, } 30-60 \text { min, }>60 \\
\text { min } \\
\text { Pedometer-manual or automatic } \\
\text { synchronisation? } \\
\text { Number of steps per day } \\
\text { Number of activity minutes reached } \\
\text { per day } \\
\text { Activities selected on a given day }\end{array}$ \\
\hline
\end{tabular}

\section{Data management}

All collected primary data will be processed under strict confidentiality and will not be made accessible to third parties. Questionnaire data are collected via SURVEY (https://bildungsportal.sachsen.de/umfragen/). This service is based on Limesurvey and is provided by BPS Bildungsportal Sachsen GmbH. When using it, the following privacy policy applies (https://bildungsportal. sachsen.de/umfragen/datenschutz.html). The app data of 'VIDEA bewegt' is stored on the servers of TUMAINI. Members of the research team will have access to both data sets. In order to be able to contact app users, their email addresses must first be transferred from 'VIDEA bewegt' to a participant table in SURVEY. At both points of data analysis (T2 and T6) app-internal data will be transferred. This allows for the connection and anonymisation of both data sets. Data collection, coding and database cleaning is performed by at least two researchers (authors). A plausibility check is performed by descriptive univariate analysis. A university email address is provided to participants in case of questions or problems. A data sharing plan was added to the trial registration.

\section{Statistical methods}

Sociodemographic data and user behaviour will be analysed descriptively.

The effect on outcomes is calculated using a twosided t-test for dependent samples at $\mathrm{T} 1$ to $\mathrm{T} 6$. The t-test requires metric, normally distributed variables. ${ }^{49}$ The Kolmogorov-Smirnov test will be used to test for normal distribution. In case of normal distribution, the connected t-test will be used, if there is no normal distribution the Wilcoxon sign rank test. In each case, the CI $(95 \%)$ is tested with $\mathrm{p}=0.05$ (see table 3 ). The possible alpha-error inflation of multiple mean value comparisons is controlled by Bonferroni correction. In addition, a power analysis using $\mathrm{G}^{*}$ Power $^{39}$ is performed, the CI for significance estimation is determined and the Bayesian correction is applied. ${ }^{50}$

There is no agreed definition of a general clinical relevance margin for increase of daily step count, as these improvement rates differ for certain patient characteristics (like sex, diseases and the baseline level of physical activity). ${ }^{51}$ In earlier trials, the clinical importance of change in step count (as one of the primary outcomes in our study) was considered as 600 and $1100^{52} 53$ and above $^{5455}$ in different chronic diseases.

In the light of our relatively broad inclusion criteria, effects on primary and secondary outcomes will be interpreted with caution and in strong accordance to the enrolled patient profiles.

\section{Methods for any additional analyses (e.g. subgroup and adjusted analyses)}

A multiple regression analysis (possibly multilevel models) is planned. Predictors are age, gender, body mass index, baseline activity, participation in further lifestyle interventions. 
Table 3 Statistical methods

\begin{tabular}{|c|c|c|}
\hline Outcome & Hypothesis & Statistical analysis \\
\hline \multicolumn{3}{|l|}{ Primary outcome } \\
\hline $\begin{array}{l}\text { Average number of steps/activity- } \\
\text { minutes per day }\end{array}$ & $\begin{array}{l}\text { Increase within first } 4 \text { weeks and until } \\
\text { programme completion compared with } \\
\text { baseline }\end{array}$ & $\begin{array}{l}\text { t-test for combined samples (or } \\
\text { Wilcoxon test) }\end{array}$ \\
\hline \multicolumn{3}{|l|}{ Secondary outcomes } \\
\hline Average MET-minutes per week & $\begin{array}{l}\text { Increase within first } 4 \text { weeks and until } \\
\text { programme completion compared with } \\
\text { baseline, maintained over } 2 / 4 / 6 / 12 \text { months }\end{array}$ & t-test (or Wilcoxon-test) \\
\hline $\begin{array}{l}\text { Self-efficacy (motivational, maintaining, } \\
\text { restoring self-efficacy) }\end{array}$ & $\begin{array}{l}\text { Increase within first } 4 \text { weeks and until } \\
\text { programme completion compared with } \\
\text { baseline, maintained over } 2 / 4 / 6 / 12 \text { months }\end{array}$ & t-test (or Wilcoxon-test) \\
\hline $\begin{array}{l}\text { Self-assessment endurance, muscle } \\
\text { strength }\end{array}$ & $\begin{array}{l}\text { Increase within first } 4 \text { weeks and until } \\
\text { programme completion compared with } \\
\text { baseline, maintained over } 2 / 4 / 6 / 12 \text { months }\end{array}$ & t-test (or Wilcoxon-test) \\
\hline $\begin{array}{l}\text { User behaviour (Peva-FB after } \\
\text { completion of the programme, or in } \\
\text { case of dropout, additional continuously } \\
\text { recorded internal system data (see } \\
\text { table 1)) }\end{array}$ & & $\begin{array}{l}\text { Descriptive analysis with } \\
\text { frequencies, mean values, SD }\end{array}$ \\
\hline $\begin{array}{l}\text { Subgroup analysis } \\
\text { (eg, age, gender, BMI, baseline activity, } \\
\text { participation in additional lifestyle } \\
\text { interventions) }\end{array}$ & & $\begin{array}{l}\text { Multiple linear regression } \\
\text { (multilevel model, if applicable) }\end{array}$ \\
\hline
\end{tabular}

BMI, body mass index; MET, metabolic equivalent; Peva, process evaluation; SF-8, Short Form Health Survey 8.

\section{Auditing}

A pretest of the questionnaires took place before starting data collection.

Additionally, system-internal data collection is checked using test accounts.

\section{DISCUSSION}

The main aim of the described study is to assess the effectiveness of the video-based smartphone app 'VIDEA bewegt' for increasing physical activity in German adults under real-life conditions. To the best of our knowledge, 'VIDEA bewegt' is one of the first apps on the German market to offer a primary preventative course to increase physical activity being developed under the guidance of physicians and being certified and covered by health insurances. Although the potential of digital prevention programmes is very promising, ${ }^{9} 1718$ very few apps available on the market have yet been scientifically evaluated. ${ }^{23}$ In order to increase the clinical benefit of digital interventions, scientific analysis is urgently needed..$^{25}$

Data are collected using various methods. The app system provides synchronised step numbers and activity minutes while established questionnaires allow for further data collection.
Since the acceptance of technical innovations substantially depends on ease of use and perceived usefulness, ${ }^{56}$ a usability test was carried out as the basis of this study. Previous studies have shown the importance of usability testing for successful app development. ${ }^{57-59}$ The thinkaloud method has proven to be a useful way to gain insight into the thought processes and problems of test persons, especially in digital health interventions. ${ }^{31}{ }^{58}$ In order to apply the results of the usability test, a process evaluation is included in the study, in which usage data of the app, as well as obstacles during app use, will be evaluated.

The development of the study protocol followed important recommendations formulated by Eysenbach and the Consolidated Standards of Reporting TrialsEHEALTH Group. ${ }^{60}$ As such, we describe the development of the video-based app and how to access it and provide detailed information on the intervention, including the mode of delivery, features/functionalities/ components and promts/reminders used.

In summary, the described study aims to contribute to exploring the potential of digital interventions for effectively increasing physical activity.

\section{Limitations and strengths}

The described study design contains a series of limitations. 
First of all, there is a risk of selection bias. Since participation in the evaluation is voluntary, it is reasonable to assume that people who are already highly motivated to increase their level of physical activity are going to show more interest in participating than users who are struggling with their awareness and motivation. Additionally, it is planned to offer financial incentive to participate in the study by waiving the otherwise applicable course fee, which may bias the results. As both healthy subjects, as well as individuals at risk for and those already living with chronic disease are addressed, inclusion criteria are broad. This may result in an inhomogeneous group of study participants, which is also influenced by characteristics like age, frequency/intensity of smartphone use or level of health literacy.

Due to the multilayered structure of the intervention with several components, the actual intervention will differ for each individual (risk of performance bias). This study can only collect limited data on how intensively the individual components of the app have been used and how they contributed to the overall effect.

The app is regularly updated by the responsible company. The evaluation team does not have any influence on these updates but will be informed via email in case an update has been conducted. However, these updates mostly intent to eliminate technical problems and do not lead to major changes of the overall intervention.

Most of the data collected is based on self-assessment, there is no possibility of verification. The study is not conducted as a randomised controlled trial, there will be no initial trial visit and no in-person contact during the intervention. For this reason, no blinding of the participants and the study personnel is intended. The design of the study resembles a pragmatic study. ${ }^{62}$ Due to the pragmatic approach measuring precise baseline data are difficult. Since the users will need to register in the app before participating in the study, the participants' level of knowledge may differ by the time the first questionnaire is answered.

These limitations are countered by the strength of the described study to evaluate the effectiveness of the app under the most realistic conditions possible. The project includes several different approaches, such as a usability test, questionnaires, system data and a follow-up to assess sustainability. The aim is to create a comprehensive impression of the app, its weaknesses, strengths and potential.

\section{ETHICS AND DISSEMINATION \\ Ethics approval}

After an ethics application was submitted to the Ethics Committee of the Technical University of Dresden on 12 April, the Ethics Committee approved the study on 25 May 2019 (EK 272062019).

\section{Protocol amendments}

If changes to the procedure are necessary, a report will be made to the ethics committee of the Technical University of Dresden as well as to the study participants.

\section{Consent to participate}

The informed consent to participate is obtained written by clicking a button in the first online questionnaire in accordance with the General Data Protection Regulation (GDPR) as a prerequisite for participation (figure 1).

\section{Dissemination policy}

The results of the study are planned to be published in a scientific journal.

Contributors TF, GR and PS conceived and designed the study protocol, wrote the manuscript, will collect the data and perform the data analysis. PT regularly provided feedback on the overall study protocol, was involved in the development of the usability test, and participated in the writing of the manuscript. PEHS advised and provided feedback on the overall protocol and reviewed the manuscript. All authors reviewed and approved the final version of the manuscript before submission.

Funding The authors have not declared a specific grant for this research from any funding agency in the public, commercial or not-for-profit sectors. The TUMAINI Institut für Präventions management GmbH provides necessary system-internal data. We acknowledge support by the Open Access Publication Funds of the SLUB/ TU Dresden.

Competing interests The principal investigator PEHS was involved in the development and implementation of the app 'VIDEA bewegt' as a medical expert. $\mathrm{He}$ is responsible for the medical and theoretical background and is shown in the app's videos. He received no payment for his participation in the app.

Patient consent for publication Not required.

Provenance and peer review Not commissioned; externally peer reviewed.

Open access This is an open access article distributed in accordance with the Creative Commons Attribution Non Commercial (CC BY-NC 4.0) license, which permits others to distribute, remix, adapt, build upon this work non-commercially, and license their derivative works on different terms, provided the original work is properly cited, appropriate credit is given, any changes made indicated, and the use is non-commercial. See: http://creativecommons.org/licenses/by-nc/4.0/.

ORCID iD

Tillmann Fischer http://orcid.org/0000-0003-3411-9838

\section{REFERENCES}

1 GBD 2015 Risk Factors Collaborators. Global, regional, and national comparative risk assessment of 79 behavioural, environmental and occupational, and metabolic risks or clusters of risks, 1990-2015: a systematic analysis for the global burden of disease study 2015 . Lancet 2016;388:1659-724.

2 Hallal PC, Andersen LB, Bull FC, et al. Global physical activity levels: surveillance progress, pitfalls, and prospects. Lancet 2012;380:247-57.

$3 \mathrm{WHO}, \mathrm{GHO}$. Prevalence of insufficient physical activity among adults - Data by WHO region, 2016. Available: http://apps.who.int/gho/data/ view.main.2482?lang=en [Accessed 24 Mar 2019].

4 Buchman AS, Wilson RS, Yu L, et al. Total daily activity declines more rapidly with increasing age in older adults. Arch Gerontol Geriatr 2014;58:74-9.

5 Warburton DER, Nicol CW, Bredin SSD. Health benefits of physical activity: the evidence. CMAJ 2006;174:801-9.

6 Lee I-M, Shiroma EJ, Lobelo F, et al. Effect of physical inactivity on major non-communicable diseases worldwide: an analysis of burden of disease and life expectancy. Lancet 2012;380:219-29.

7 Fiuza-Luces C, Garatachea N, Berger NA, et al. Exercise is the real polypill. Physiology 2013;28:330-58.

8 Mosa ASM, Yoo I, Sheets L. A systematic review of healthcare applications for smartphones. BMC Med Inform Decis Mak 2012;12:67.

9 Safran Naimark J, Madar Z, Shahar DR. The impact of a webbased APP (eBalance) in promoting healthy lifestyles: randomized controlled trial. J Med Internet Res 2015;17:e56.

10 Goyal S, Cafazzo JA. Mobile phone health apps for diabetes management: current evidence and future developments. QJM 2013;106:1067-9. 
11 Haas M. Smartphone-Markt: Konjunktur und trends. Bitkom, 2018.

12 Romeo A, Edney S, Plotnikoff R, et al. Can smartphone Apps increase physical activity? systematic review and meta-analysis. $J$ Med Internet Res 2019;21:e12053.

13 Lee M, Lee H, Kim Y, et al. Mobile App-Based health promotion programs: a systematic review of the literature. Int J Environ Res Public Health 2018;15. doi:10.3390/ijerph15122838. [Epub ahead of print: 13 Nov 2018].

14 Schoeppe S, Alley S, Van Lippevelde W, et al. Efficacy of interventions that use apps to improve diet, physical activity and sedentary behaviour: a systematic review. Int J Behav Nutr Phys Act 2016;13:127.

15 Glynn L, Hayes PS, Casey M, et al. Effectiveness of a smartphone application to promote physical activity in primary care: the smart move randomised controlled trial. Br J Gen Pract 2014:384-91.

16 Albrecht U, Kurzfassung K. Chancen und Risiken von GesundheitsApps (CHARISMA). Medizinische Hochschule Hannover, 2016.

17 Dallinga JM, Mennes M, Alpay L, et al. App use, physical activity and healthy lifestyle: a cross sectional study. BMC Public Health 2015;15:833.

18 Harries T, Eslambolchilar P, Rettie R, et al. Effectiveness of a smartphone APP in increasing physical activity amongst male adults: a randomised controlled trial. BMC Public Health 2016;16:1-10.

19 Knight E, Stuckey MI, Prapavessis $\mathrm{H}$, et al. Public health quidelines for physical activity: is there an APP for that? A review of android and apple APP stores. JMIR Mhealth Uhealth 2015;3:e43.

20 Heath GW, Parra DC, Sarmiento OL, et al. Evidence-Based intervention in physical activity: lessons from around the world. Lancet 2012;380:272-81.

21 Stuckey MI, Carter SW, Knight E. The role of smartphones in encouraging physical activity in adults. Int $J$ Gen Med 2017;10:293-303.

22 Zhao J, Freeman B, Li M. Can mobile phone Apps influence people's health behavior change? an evidence review. J Med Internet Res 2016;18:e287.

23 Coughlin SS, Whitehead M, Sheats JQ, et al. A review of smartphone applications for promoting physical activity. Jacobs $J$ Community Med 2016;2. [Epub ahead of print: 11 Feb 2016].

24 Payne HE, Lister C, West JH, et al. Behavioral functionality of mobile apps in health interventions: a systematic review of the literature. JMIR Mhealth Uhealth 2015;3:e20.

25 Jake-Schoffman DE, Silfee VJ, Waring ME, et al. Methods for evaluating the content, usability, and efficacy of commercial mobile health Apps. JMIR Mhealth Uhealth 2017;5:e190.

26 Neugebauer EAM, Rath A, Antoine S-L, et al. Specific barriers to the conduct of randomised clinical trials on medical devices. Trials 2017;18:427.

27 Müller G, Weser G, Schwarz PEH. The European perspective of diabetes prevention: the need for individualization of diabetes prevention. J Endocrinol Invest 2013;36:352-7.

28 Hanlon P, Daines L, Campbell C, et al. Telehealth interventions to support self-management of long-term conditions: a systematic metareview of diabetes, heart failure, asthma, chronic obstructive pulmonary disease, and cancer. J Med Internet Res 2017;19:e172-26.

29 Cui M, Wu X, Mao J, et al. T2Dm self-management via smartphone applications: a systematic review and meta-analysis. PLoS One 2016;11:e0166718.

30 Alpert JM, Krist AH, Aycock RA, et al. Designing User-Centric patient portals: clinician and patients' uses and Gratifications. Telemed J E Health 2017;23:248-53.

31 Årsand E, Demiris G. User-centered methods for designing patientcentric self-help tools. Inform Health Soc Care 2008;33:158-69.

32 Jordan S, McSwiggan J, Parker J, et al. An mHealth APP for decision-making support in wound dressing selection (wounds): protocol for a User-Centered feasibility study. JMIR Res Protoc 2018;7:e108.

33 Esser PE, Goossens RHM. A framework for the design of usercentred teleconsulting systems. J Telemed Telecare 2009;15:32-9.

34 Wildenbos GA, Peute L, Jaspers M. Aging barriers influencing mobile health usability for older adults: a literature based framework (MOLDUS). Int J Med Inform 2018;114:66-75.

35 VIDEA bewegt. Available: https://videabewegt.de [Accessed 27 Nov 2019].

36 Greaves CJ, Reddy P, Sheppard K. Supporting behaviour change for diabetes prevention. Diabetes Prev Pract 2010.

37 Lubans DR, Smith JJ, Skinner G, et al. Development and implementation of a smartphone application to promote physical activity and reduce screen-time in adolescent boys. Front Public Health 2014;2:42.
38 Martin CK, Miller AC, Thomas DM, et al. Efficacy of SmartLoss, a smartphone-based weight loss intervention: results from a randomized controlled trial. Obesity 2015;23:935-42.

39 Faul F, Erdfelder E, Buchner A, et al. Statistical power analyses using $G^{*}$ Power 3.1: tests for correlation and regression analyses. Behav Res Methods 2009;41:1149-60.

40 French DP, Olander EK, Chisholm A, et al. Which behaviour change techniques are most effective at increasing older adults' self-efficacy and physical activity behaviour? A systematic review. Ann Behav Med 2014:48:225-34.

41 Williams SL, French DP. What are the most effective intervention techniques for changing physical activity self-efficacy and physical activity behaviour--and are they the same? Health Educ Res 2011;26:308-22.

42 Olander EK, Fletcher $\mathrm{H}$, Williams S, et al. What are the most effective techniques in changing obese individuals' physical activity selfefficacy and behaviour: a systematic review and meta-analysis. Int $J$ Behav Nutr Phys Act 2013;10:29.

43 Maxwell SE, Appelbaum MI. Sample size and multiple regression analysis. Psychol Methods 2000;5:434-58.

44 Maxwell SE, Kelley K, Rausch JR. Sample size planning for statistical power and accuracy in parameter estimation. Annu Rev Psychol 2008:59:537-63.

45 Bolle S, Romijn G, Smets EMA, et al. Older cancer patients' user experiences with web-based health information tools: a Think-Aloud study. J Med Internet Res 2016;18:e208.

46 Rubin J, Chisnell D. Handbook of usability testing, 2008.

47 Nielsen J. Estimating the number of subjects needed for a thinking aloud test. Int J Hum Comput Stud 1994;41:385-97.

48 Forum Qualitative Sozialforschung. Forum: qualitative social research 2000.

49 Bortz J, Schuster C. Statistik für human- und Sozialwissenschaftler, 2010.

50 Dienes Z. Using Bayes to get the most out of non-significant results. Front Psychol 2014;5:781.

51 Dwyer T, Hosmer D, Hosmer T, et al. The inverse relationship between number of steps per day and obesity in a population-based sample - the AusDiab study. Int J Obes 2007;31:797-804.

52 Motl RW, Pilutti LA, Learmonth YC, et al. Clinical importance of steps taken per day among persons with multiple sclerosis. PLoS One 2013;8:e73247.

53 Demeyer H, Burtin C, Hornikx M, et al. The minimal important difference in physical activity in patients with COPD. PLOS One 2016;11:e0154587.

54 Motl RW, Dlugonski D. Increasing physical activity in multiple sclerosis using a behavioral intervention. Behav Med 2011:37:125-31.

55 Dlugonski D, Motl RW, McAuley E. Increasing physical activity in multiple sclerosis: replicating Internet intervention effects using objective and self-report outcomes. J Rehabil Res Dev 2011;48:1129-36.

56 Harst L, Lantzsch H, Scheibe M. Theories predicting end-user acceptance of telemedicine use: systematic review. J Med Internet Res 2019;21:e13117.

57 O'Malley G, Dowdall G, Burls A, et al. Exploring the usability of a mobile APP for adolescent obesity management. JMIR Mhealth Uhealth 2014;2:e29.

58 Jaspers MWM. A comparison of usability methods for testing interactive health technologies: methodological aspects and empirical evidence. Int J Med Inform 2009;78:340-53.

59 Brown W, Yen P-Y, Rojas M, et al. Assessment of the health it usability evaluation model (Health-ITUEM) for evaluating mobile health (mHealth) technology. J Biomed Inform 2013;46:1080-7.

60 Eysenbach G. CONSORT-EHEALTH: improving and standardizing evaluation reports of web-based and mobile health interventions. $J$ Med Internet Res 2011;13:e126.

61 Ford I, Norrie J, Drazen JM. Pragmatic trials. N Engl J Med 2016;375:454-63.

62 Loudon K, Treweek S, Sullivan F, et al. The PRECIS-2 tool: designing trials that are fit for purpose. BMJ 2015;350:h2147.

63 Armstrong T, Bull F. Development of the world Health organization global physical activity questionnaire (GPAQ). J Public Health 2006;14:66-70.

64 Wanner M, Hartmann C, Pestoni G, et al. Validation of the global physical activity questionnaire for self-administration in a European context. BMJ Open Sport Exerc Med 2017;3:e000206.

65 World Health Organisation (WHO). GPAQ analysis guide, 2005: 46-9.

66 Ellert U, Lampert T, Ravens-Sieberer U. Messung Der gesundheitsbezogenen Lebensqualität MIT dem SF-8. Bundesgesundheitsblatt - Gesundheitsforsch - Gesundheitsschutz 2005;12:1330-7. 
67 Ware JE, Kosinski M, Dewey JE. How to score and interpret singleitem health status measures: a manual for users of the SF-8TM Health Survey. Medicine 2001.

68 Burkert S, Scholz U, Gralla O, et al. Dyadic planning of healthbehavior change after prostatectomy: a randomized-controlled planning intervention. Soc Sci Med 2011;73:783-92.
69 Sniehotta FF, Scholz U, Schwarzer R. Action plans and coping plans for physical exercise: a longitudinal intervention study in cardiac rehabilitation. Br J Health Psychol 2006;11:23-37.

70 Schrepp M, Hinderks A, Thomaschewski Jörg. Design and evaluation of a short version of the user experience questionnaire (UEQ-S). IJIMAI 2017;4:103. 